\title{
REVISIÓN
}

\section{Enfoque teórico del crecimiento físico de niños y adolescentes}

\author{
Rossana Gomez-Campos ${ }^{a, b}$,Miguel Arruda ${ }^{a}$, Cristian Luarte-Rocha ${ }^{c}$, Camilo Urra Albornoz ${ }^{d}$, \\ Alejandro Almonacid Fierro 'b Marco Cossio-Bolaños ${ }^{e, f, *}$
}

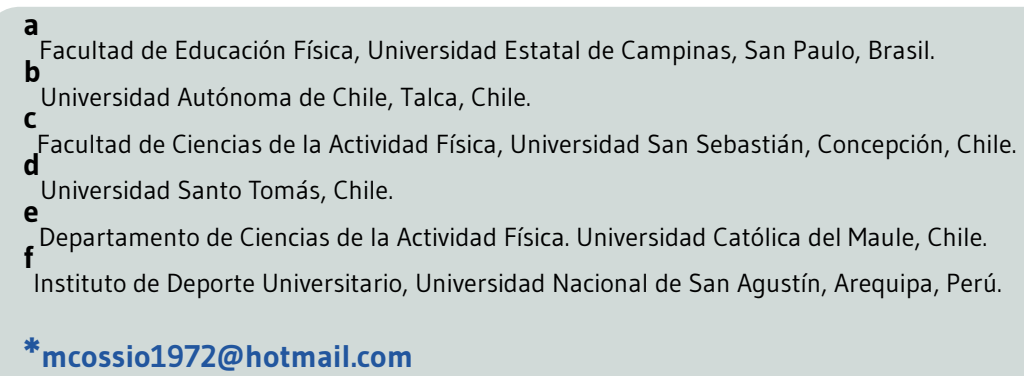

Recibido el 30 de noviembre de 2015; aceptado el 20 de junio de 2016.

Enfoque teórico del crecimiento físico de niños y adolescentes

\section{PALABRAS CLAVE}

Crecimiento;

Pesos y medidas corporales;

Desarrollo infantil; Lactante; Niño; Adolescente.

\section{RESUMEN}

El estudio del crecimiento físico permite comprender la variabilidad de este proceso en el ser humano de acuerdo con su potencial genético, características biológicas y factores ambientales. Factores extrínsecos como intrínsecos, podrían afectar el normal proceso de crecimiento físico, los que son estudiados a través de diferentes tipos de investigaciones, constituyéndose en una interesante temática para los profesionales de las ciencias de la salud. El objetivo del estudio es analizar los factores que afectan el crecimiento físico y describir los tipos de investigación transversal, longitudinal y ex post facto utilizados en el estudio del crecimiento físico. Los factores intrínsecos, medioambientales y la interacción entre ambos, deberían ser abordados en diversas poblaciones, priorizando los posibles factores que afectan al crecimiento físico de niños y adolescentes. Esto permitirá diagnosticar, clasificar y monitorizar el crecimiento físico en función del tiempo (transversal y longitudinal) y retrospectiva (ex post facto); consecuentemente surgirá la posibilidad para que investigadores y/o instituciones privadas y gubernamentales promuevan la realización de estudios antropométricos validados que puedan contribuir a ejercer políticas sanitarias eficaces, para diversos fines como son el exceso de peso en la edad infantil y otras entidades nosológicas relacionadas con la nutrición. 
Theoretical approach of physical growth of children and adolescents

\section{KEYWORDS}

Growth;

Body Weights and

Measures;

Child Development;

Infant;

Child;

Adolescent

\section{ABSTRACT}

According to their genetic potential, biological characteristics and environmental factors, the study of physical growth allows us to understand the variability of this process in humans. Extrinsic and intrinsic factors could affect the normal process of physical growth, which are studied through different types of research, becoming an interesting subject for professionals in the health sciences. The aim of this study is to analyze the factors affecting physical growth and describe the types of research (transverse, longitudinal and ex post facto) used in the study of physical growth. Intrinsic factors, environmental factors and the interaction between them should be studied in diverse population prioritizing the potential factors affecting physical growth of children and adolescents. This will diagnose, classify and monitor physical growth versus time (transverse and longitudinal) and retrospectively (ex post facto). Consequently, the possibility for researchers and/or private and government institutions arises to promote validated anthropometric studies, helping to implement effective health policies for various purposes such as excess weight control in childhood and other disease entities related nutrition.

\section{CITA}

Gomez-Campos R, Arruda M, Luarte-Rocha C, Urra Albornoz C, Almonacid Fierro A, Cossio-Bolaños M. Enfoque teórico del crecimiento físico de niños y adolescentes. Rev Esp Nutr Hum Diet. 2016; 20(3): 244 - 253. doi: 10.14306/ renhyd.20.3.198

\section{INTRODUCCIÓN}

El estudio del crecimiento físico se remonta al siglo XVIII, donde la antropometría empezó a ser utilizada para medir la estatura en niños, pasando posteriormente a cuantificar el cuerpo humano, lo que permitió que en Europa se adoptaran políticas nacionales de salud.

Formalmente ha sido estudiada hace más de 170 años, y con el apoyo de la ciencia se ha logrado comprender la variabilidad del crecimiento físico humano de acuerdo con su potencial genético, características biológicas y factores ambientales. De hecho, son muchos los cambios que se han producido hasta la actualidad, sobre todo en relación a los factores extrínsecos, los que a menudo son estudiados por investigaciones de tipo transversal, longitudinal y ex post facto.

Básicamente el estudio del crecimiento físico, el desarrollo y el bienestar, están directamente relacionados con el estado nutricional ${ }^{1}$, lo que implica el uso de estándares referidos a criterios y/o normas para su valoración, diagnóstico, moni- torización y clasificación de individuos y grupos. En ese sentido, son varios los estándares que existen en el mundo ${ }^{2-5}$, sin embargo, la Organización Mundial de la Salud (OMS) sugiere la adopción de sus tablas de crecimiento, en especial en aquellos lugares donde no existe ninguna normativa ${ }^{6}$, a pesar de que no reflejan los patrones de crecimiento físico de determinadas poblaciones. Utilizar muestras descriptivas de poblaciones que reflejan una tendencia a largo plazo hacia el sobrepeso y la obesidad para establecer patrones de crecimiento conduce involuntariamente a un no deseable sesgo al alza que lleva a subestimar el sobrepeso y la obesidad, así como a sobreestimar la desnutrición ${ }^{4}$.

Desde esa perspectiva, estudiar el crecimiento físico desde un enfoque teórico es relevante, puesto que en la literatura son escasos los estudios de revisión bibliográfica que permiten analizar y estudiar el crecimiento físico de niños y adolescentes, constituyéndose en una interesante temática para los profesionales de las ciencias de la salud. Por lo tanto, el objetivo del estudio fue analizar los factores que afectan al crecimiento físico y describir los tipos de investigación transversal, longitudinal y ex post facto utilizados en su estudio. 


\section{METODOLOGÍA}

La estrategia utilizada, basada en la búsqueda bibliográfica efectuada del 20 de noviembre de 2014 al 31 de enero de 2015, permitió localizar las publicaciones posteriores al año 1995 de las bases de datos on-line más importantes en el área de la Salud y la Educación Física, incluyendo: PubMedMedline, Scopus, Scielo, LILACS, Sport-Discus. Como criterios de inclusión se consideraron: a) estudios de revisión; b) estudios descriptivos; y c) estudios de intervención. Los criterios de exclusión fueron: a) estudios publicados en forma de resumen o comunicaciones cortas; y b) redactados en idioma distinto al inglés, español o portugués. Como palabras clave se utilizaron: crecimiento físico, estándares, factores, valoración.

\section{ENFOQUE TEÓRICO DEL CRECIMIENTO FÍSICO}

\section{Aspectos conceptuales}

Clásicamente, el crecimiento físico ha sido definido como la secuencia de modificaciones somáticas que sufre un organismo biológico, que se explica, como el aumento en el número o tamaño de las células que componen los diversos tejidos del organismo. Así, Malina y Bouchard explican que este fenómeno es fruto de la hiperplasia, hipertrofia y la agregación, y que al presentar períodos de aceleración y desaceleración, hacen necesario llevar en consideración las variaciones individuales en función de la edad y sexo.

Desde el punto de vista fisiológico, el crecimiento lineal se constituye sobre la infraestructura esquelética; consecuentemente, la fusión de la epífisis y las metáfisis de los huesos largos caracterizan la terminación del proceso de crecimiento físico. En términos antropométricos, consiste en el aumento y modificación de los componentes corporales, tanto longitudinal, como transversalmente ${ }^{8}$.

En general, el crecimiento físico tiene dos etapas, la prenatal (tiene lugar intraútero desde el primer instante de la concepción y acaba con el nacimiento) y la postnatal que ocurre en tres fases: infancia, niñez y adolescencia. En esta última fase, a su vez, se describen otras tres: adolescencia temprana, generalmente entre los 12 y 13 años de edad; adolescencia media, entre los 14 y 16 años de edad; y adolescencia tardía, entre los 17 y 21 años de edad. En todas ellas se crean oportunidades para que el individuo pueda expresar su potencial genético, pero a su vez, este puede ser modificado por el medio ambiente ${ }^{9}$, lo que conlleva un constante control, diagnóstico y seguimiento del proceso de crecimiento con el objetivo de identificar niños con riesgo nutricional.

\section{Tipos de crecimiento físico}

En general, ocurren básicamente cuatro tipos principales de crecimiento en los diferentes órganos y tejidos (Figura 1$)^{10}$. Estos tipos de crecimiento son valorados e interpretados a través de la curva propuesta en el año 1930 por Scammon ${ }^{10}$, donde a través de porcentajes se expresa la evolución del crecimiento somático (general), neural, genital y linfoide.

El crecimiento general, somático y/o patrón, se refiere al crecimiento total de las dimensiones externas del cuerpo, incluyendo el esqueleto, con excepción de la cabeza y el cue$\mathrm{Ilo}^{7}$; así el crecimiento del tejido muscular, óseo, volumen sanguíneo, órganos del aparato respiratorio, circulatorio, digestivo, riñones y bazo son expresados en mediciones antropométricas que incluyen gráficas y tablas de diversas variables: peso, estatura, envergadura o braza, perímetros braquiales, de cintura, de cadera, de muslo y de pantorrilla, longitud de las extremidades, pliegues cutáneos, etc.

El crecimiento neural (cerebral y cefálico) incluye las dimensiones cefálicas (excluyendo el área facial), refiriéndose al crecimiento del encéfalo, cerebelo, estructuras asociadas a

Figura 1. Tipos de crecimiento físico. Tomado de Scammon ${ }^{10}$.

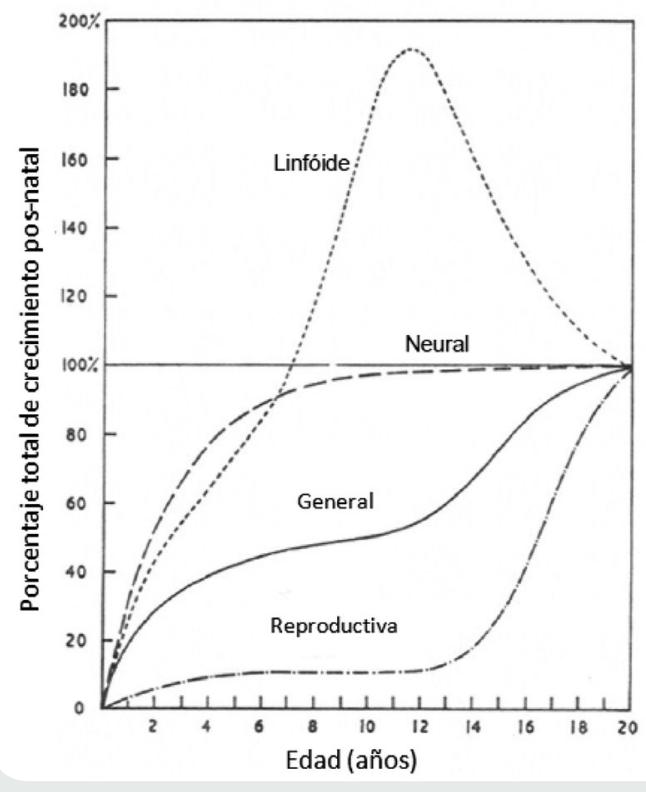


los órganos de la visión, oído y partes relacionadas al cráneo, los cuales se desarrollan a una velocidad superior al resto del cuerpo ${ }^{7}$. La intensa velocidad de crecimiento observada en los dos primeros años de vida ${ }^{11}$ está representada por el perímetro cefálico que aumenta alrededor de $20 \mathrm{~cm}$ desde el nacimiento hasta los 18 meses.

Finalmente, el crecimiento linfoide y genital (reproductivo), describen el crecimiento de las glándulas relacionadas con el sistema inmunológico y las características sexuales primarias y secundarias, respectivamente.

\section{Factores que influyen en el crecimiento físico}

Hace más de 185 años se alertó sobre la posibilidad de intervención de factores extrínsecos sobre el crecimiento físico, lo que fue observado en los soldados del ejército de Napoleón cuando fueron clasificados según su procedencia de barrios más pobres o alejados.

Actualmente, es aceptado que el crecimiento físico está condicionado por la herencia genética, pero es fuertemente influenciado por el ambiente físico ${ }^{12}$ y social, principalmente en lo relacionado a las enfermedades y al estado nutricional ${ }^{13}$; aunque factores culturales, como el tipo de alimentación, higiene ${ }^{14}$, actividad física y la tendencia secular ${ }^{15}$ influyen sobre el proceso de crecimiento físico en diversas regiones del mundo (Figura 2).

Factores intrínsecos: Se considera los factores relacionados al sistema Neuro-endocrino ${ }^{16}$, explicando que el factor genético determina la adquisición de una estatura que

Figura 2. Factores que afectan el crecimiento físico.

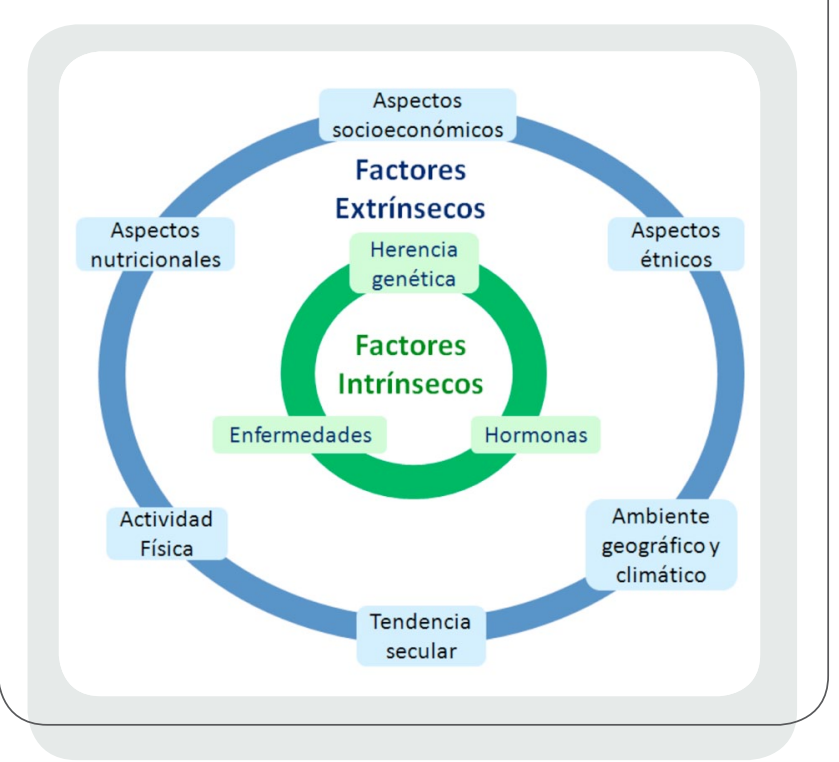

represente el $100 \%$ de su capacidad de crecimiento y al existir disminución del material genético, exceso o expresión anormal, la estatura será menor a la esperada. Aunque, el impacto del factor genético puede ser afectado por factores extrínsecos ${ }^{17}$, la persistencia de situaciones adversas puede impedir que los niños alcancen su máximo potencial genético. Dentro de estos factores destacamos: la herencia genética, en razón que el factor genético que se encuentra en el cigoto, es el que determina las potencialidades máximas para la estatura, peso, longitud de miembros, estructuras óseas y aspectos faciales ${ }^{18}$; las hormonas, debido a que las instrucciones bioquímicas que dependen de las funciones neural y hormonal afectan los diferentes órganos y sistemas corporales, de esa forma, el sistema endócrino actúa en el mecanismo que unifica la interacción de los genes y el ambiente; y finalmente, la presencia de enfermedades es otro factor que ocasiona una velocidad de crecimiento anormal, diminución de la relación peso/estatura y atraso de la edad ósea en relación a la edad cronológica.

Factores extrínsecos: Las condiciones del medio ambiente podrían afectar los dominios biológicos y comportamentales. Entre ellas se destacan:

- Los aspectos socioeconómicos, que actúan como factores de riesgo directos o indirectos que afectan al estado nutricional infantil e influyen sobre los hábitos alimentarios. En este aspecto, la renta percápita y familiar están relacionadas con factores como la educación, tipo de vivienda, servicios de sanidad, acceso a los servicios de salud y bienes de consumo ${ }^{19}$; de esa forma, surge una relación indirecta entre la disponibilidad de comida rápida y la obesidad, que se explica por el aumento en el consumo de comida rápida y alimentos procesados de bajo contenido nutricional, que generan alteraciones en los patrones de alimentación tradicionales, aumentando la prevalencia de sobrepeso y obesidad.

- Entre los factores étnicos, el estilo de vida, la dieta, educación y la vivienda son culturalmente determinados por los patrones de hábitos, aptitudes y comportamientos, generando diferencias de crecimiento entre los grupos étnicos. Diversas investigaciones han establecido que las conductas sedentarias y la falta de actividad física están en parte determinadas por factores de tipo familiar ${ }^{20}$.

- Otro aspecto, como el nutricional, es prioritario para el cumplimiento de las funciones fisiológicas, así como para el propio proceso de crecimiento físico ${ }^{21}$; por lo que la subalimentación provocaría alteraciones irrecuperables sobre el crecimiento físico y una hiperalimentación ocasionaría condiciones de sobrepeso y obesidad. 
- Por otro lado, la actividad física realizada regularmente favorece un crecimiento normal y mantiene la integridad de los tejidos; sin embargo, la literatura también ha alertado sobre los posibles efectos negativos que ocasionaría la carga de entrenamiento excesiva sobre los jóvenes, por los riesgos asociados a la práctica intensa y poco preparada ${ }^{22}$ que podría afectar consecuentemente al crecimiento y a la maduración ${ }^{23}$.

- Entre los factores geográficos, Frisancho ${ }^{24}$ destaca que habitantes de zonas asociadas a una baja temperatura, escasa humedad relativa, alta radiación solar e hipoxia, generalmente presentan un crecimiento lento y prolongado que se traduce en un menor tamaño corporal en la edad adulta, siendo más pequeños que los habitantes de zonas cercanas al nivel del mar, que además poseen un tamaño y volumen torácico mayor que el de los habitantes de las tierras bajas de igual etnia y mucho mayor que el supuesto para la talla del individuo ${ }^{25,26}$. La comprensión del impacto de los cambios climáticos en temperaturas extremas de calor y frío sobre los niños es aún insipiente; aunque se ha demostrado que niños americanos que vivían en áreas tropicales tienen un menor peso corporal para la estatura de aquellos que residían en zonas temperadas ${ }^{27}$.

- Finalmente, la tendencia secular hace referencia a las modificaciones que se producen generacionalmente en el crecimiento y desarrollo de las poblaciones. Básicamente se define como una aceleración en la tendencia (positiva) y/o desaceleración de tendencia (negativa) del crecimiento y la maduración ${ }^{28}$. Desde un punto de vista unidireccional implica variaciones temporales entre generaciones y/o entre poblaciones de diferentes situaciones geográficas y socioeconómicas. Clásicamente, la tendencia secular ha buscado determinar los cambios de la edad de la menarquia y el crecimiento en altura y peso ${ }^{29}$. Actualmente hacen hincapié a la importancia del seguimiento de la prevalencia del sobrepeso y obesidad ${ }^{30}$ en determinados intervalos de tiempo, que posibilita identificar cambios no sólo en el exceso de peso, sino también en variables como la composición corporal, somatotipo, crecimiento físico, maduración biológica, consumo de alimentos, patrones de actividad física, entre otros. Existe una inclinación por las investigaciones relacionadas a la aptitud física enfocadas en la salud, rendimiento y actitud hacia los deportes en niños y adolescentes, en razón a encontrar asociación con el aumento de la incidencia de sobrepeso y obesidad ${ }^{31}$. Las influencias ambientales que ocasionan la tendencia secular positiva se caracterizan por un aumento en la disponibilidad de alimentos, y mejora de las condiciones sanitarias y de salud; de esa forma, las medidas de estatura y/o peso, estatura final alcanzada y las tasas de cambios en la estatura durante los años de crecimiento reflejan de forma exacta el estado de salud de un país y el estado nutricional medio de sus ciudadanos, siendo utilizado como un indicador de desarrollo socioeconómico. De acuerdo con esto, algunos antropólogos físicos, epidemiólogos $y$, recientemente, historiadores económicos y economistas, como Tanner ${ }^{32}$, han mostrado que la evolución de la estatura tiene bastante relación con las condiciones de vida y de la variabilidad de la especie humana dependiendo de las circunstancias históricas y los contextos socioeconómicos ${ }^{33-36}$.

\section{Valoración del crecimiento físico}

La evaluación es el proceso de delineamiento y obtención de información para posteriormente juzgar alternativas de decisión ${ }^{37}$. Cualquier evaluación se realiza contando con un parámetro de referencia y/o criterio de referencia, lo que posibilita comparar las medidas obtenidas de un sujeto con similares características a los que se pretende estudiar, y de esa manera emitir un juicio valedero y con solidez científica. En el caso específico del crecimiento físico, la técnica antropométrica es la que permite establecer patrones de vigilancia del crecimiento y desarrollo del niño y adolescente; para tal efecto, es necesario el uso de estándares referidos a normas y/o criterios. A continuación describimos cada uno de estos tipos.

Estándares referidos a normas: Se evalúa en referencia a una norma. Los resultados de un individuo o una población comúnmente se comparan con una población de referencia. Básicamente es un modelo que se define como un objetivo a alcanzar ${ }^{38}$, y sirve para establecer un nivel de normalidad. El estándar o norma por lo general refleja un crecimiento óptimo, lo que sugiere que todos los niños tienen el potencial suficiente para alcanzar ese nivel ${ }^{3}$. Los estándares por normas presentan algunas características propias, como por ejemplo, los datos deben provenir de poblaciones que presenten condiciones óptimas de bienestar, así como que la muestra debe ser determinada probabilísticamente, en consecuencia esto permitirá evitar sesgos y otorga mayor representatividad de los resultados. La OMS recomienda adoptar las tablas de crecimiento de los Estados Unidos, sobre todo donde no existe ninguna normativa ${ }^{6}$, pero evidentemente no reflejan los patrones de crecimiento de distintas poblaciones de diferentes culturas y etnias, aunque de alguna forma, sirven como un medio para diagnosticar, vigilar y monitorizar el estado de crecimiento con respecto a la población general, detectando de esta forma problemas de salud. 
Entre las curvas construidas a nivel internacional, las más usadas son: National Center for Health Statistic (NCHS) ${ }^{39}$, Center for Disease Control (CDC) $)^{2,5}$ y la de la OMS ${ }^{3,4}$.

Estándares referidos a criterios: Es un modelo que permite identificar un elevado y/o bajo riesgo para la salud. Estas referencias permiten identificar estándares mínimos para alcanzar los valores aceptables en relación a los indicadores de peso/edad, estatura/edad, peso/estatura, Índice de Masa Corporal (IMC), porcentaje de grasa, área grasa y muscular del brazo, entre otras variables.

De hecho, los estándares por criterio buscan identificar a sujetos que se encuentren suficientemente aptos como para mantener un buen estado salud en función de su edad y sexo; sin embargo, los puntos de corte son hasta la fecha materia de discusión, ya que aún no existe consenso sobre el valor de los puntos de corte en niños y adolescentes ${ }^{40}$. Estos estándares evitan la comparación entre niños y grupos, pero apuntan a identificar antropométricamente a los individuos que se encuentran dentro de los rangos normales en función de su edad y sexo.

Algunos estudios han usado el IMC para determinar el exceso de masa grasa en niños y adolescentes. Ya que los valores del IMC en niños varían en función del sexo y edad, surge la necesidad de utilizar patrones de referencia que permitan establecer los puntos de corte de sobrepeso y obesidad ${ }^{41}$. En ese contexto, Cole y Cols. ${ }^{42}$, realizaron la propuesta para uso internacional de los puntos de corte, que fueron generados por la extrapolación matemática de los valores utilizados para adultos. Pese a esto, aún no existe un consenso sobre qué referencia y puntos de corte son más adecuados, debido a que en las referencias actualizadas está siendo considerada la población que ha sufrido aumento en las últimas décadas de los valores del peso e IMC. Consecuentemente, se estaría generando una infraestimación y normalización de la obesidad infantil. En ese sentido, para evitar esos sesgos algunos estudios sugieren seguir utilizando referencias antiguas, y otros como Hormiguera ${ }^{43}$ consideran que para evaluar correctamente el riesgo cardiovascular, cada población debe establecer sus propios puntos de corte, tanto para el IMC como para la circunferencia de la cintura.

\section{Tipos de investigación para estudiar el crecimiento físico}

El objeto de estudio del crecimiento físico se centra en los posibles factores que puedan afectar al normal proceso de crecimiento (factores intrínsecos y extrínsecos). De hecho, los estudios utilizan a menudo tipos de investigación experimental, transversal longitudinal y ex post facto. En este estudio se aborda a grandes poblaciones, exceptuando las investigaciones experimentales.
Estudios transversales: Son los que permiten describir las características y cualidades de una persona o una población en un determinado momento. También son denominados estudios de prevalencia ${ }^{44}$, aunque se considera la posibilidad de establecer relaciones y asociaciones entre variables, sin buscar causalidad.

Básicamente estos estudios son utilizados para estudiar enfermedades de larga duración o cuyas manifestaciones se desarrollan lentamente, como es el caso de las enfermedades crónicas, desnutrición o mala nutrición por exceso. Presentan algunas ventajas, como el bajo costo de inversión, se aplican en un solo momento durante el tiempo y generalmente a grandes muestras, por lo que los resultados pueden ser generalizables a otros contextos (si la muestra es probabilística). Además, permiten estimar la tendencia central en cada edad, describir los parámetros del crecimiento físico y la variación de las características de un grupo de individuos, en función de la edad y sexo, posibilitan también la construcción de referencias por norma y/o por criterio para valorar y comparar el crecimiento físico y el estado nutricional, así como ser un punto de referencia para futuras comparaciones.

Estos estudios presentan algunas limitaciones, sobre todo cuando se seleccionan sujetos de forma no probabilística, lo que impide una probable generalización de los resultados. Por otro lado, no proporcionan información sobre la dinámica del proceso individual de crecimiento, así como de la experiencia práctica del evaluador(es), que cumplen un rol relevante en el proceso de recolección de datos (error técnico de medida intra e interevaluador). Algunos estudios referenciales son las curvas de $\mathrm{NCHS}^{39}$ de 3 a 18 años, CDC $^{2}$ de 0 a 20 años, $\mathrm{OMS}^{3}$ de 0 a 5 años y $\mathrm{OMS}^{4}$ de 5 a 19 años, así como las curvas brasileras de Marques y Cols. ${ }^{45}$ y Conde y Col. ${ }^{46}$.

Estudios longitudinales: Son observaciones de medidas repetidas registradas a través del tiempo, que permiten analizar los progresos; generalmente, implican más de dos mediciones a lo largo del tiempo, consecuentemente, se logra verificar la velocidad de crecimiento infantil y los cambios de tendencia secular.

Algunas de las desventajas son que estos estudios tienen alto costo, son difíciles de ejecutar, se pierde muestra durante la investigación y con frecuencia el investigador no puede controlar las circunstancias en que se obtienen las medidas repetidas. Por otro lado, son indispensables para medir la velocidad de crecimiento, la secuencia de eventos durante la pubertad, permiten la elaboración de normas de referencias y ofrecen la oportunidad de estudiar patrones individuales de cambio sobre el tiempo. Una curva de crecimiento de un estudio longitudinal es la de $\mathrm{NCHS}^{39}$ para niños de 0 a 3 años. 
Los intervalos de tiempo en que se observan y/o registran los datos se denominan puntos de tiempo u ocasiones y varían desde unos cuantos minutos a varios años. La información recolectada a intervalos regulares de tiempo permite precisar modelos de ritmo y velocidad en el crecimiento y maduración biológica, así como determinar cambios en la composición corporal, somatotipo y proporcionalidad de niños y adolescentes. Algunos autores consideran que los estudios longitudinales son más eficientes, más robustos en la selección del modelo y estadísticamente son más potentes en relación a los estudios transversales ${ }^{47,48}$. Además, Ios estudios longitudinales permiten distinguir los fenómenos a corto, medio y largo plazo.

Dentro de este grupo, se destacan los estudios de tendencia, cohorte y panel. Los de tendencia (trend) analizan cambios en una o más variables cuantitativas y/o cualitativas a través del tiempo, centrando su atención en una población determinada, pero no sobre la misma muestra de sujetos o unidades de análisis dentro de esa población, así, los sujetos del estudio durante las mediciones no son los mismos. La tendencia se logra observar a través de encuestas repetidas $^{49}$. Ejemplos clásicos son los estudios en que se observa la tendencia secular de poblaciones en peso y estatura ${ }^{32,50}$, adiposidad corporal, nivel de actividad física, entre otras variables.

Los diseños de evolución de grupo o estudios de cohorte, examinan cambios a través del tiempo en subpoblaciones y/o grupos específicos. Generalmente, los grupos están conformados por edad o época. De hecho, estos estudios se han utilizado de manera clásica para determinar la ocurrencia de un evento específico en un grupo de individuos libres del evento o enfermedad en estudio y, a menudo, conforme pasa el tiempo se retiran algunos sujetos, disminuyendo la muestra, aunque es posible que ingresen nuevos sujetos con las mismas condiciones que permitan mantener la misma cohorte. Este tipo de diseño es considerado como una herramienta esencial para el estudio de la salud, particularmente ahora que las enfermedades no transmisibles paulatinamente transforman el panorama de morbimortalidad mundial ${ }^{51}$, además de permitir delimitar diversas consecuencias o desenlaces que pueden estar asociadas con un único factor de riesgo etiológico.

Por ejemplo, Stettler y Cols. ${ }^{52}$, con el propósito de determinar si una rápida tasa de aumento de peso en la infancia temprana se asociaba con la condición de sobrepeso en la infancia, diseñó un estudio longitudinal de cohorte (desde el nacimiento hasta los 7 años), seleccionándose 27.899 participantes, nacidos entre 1959 y 1965 . Los resultados mostraron que hubo un patrón de ganancia rápida de peso durante los primeros 4 meses de vida, asociándose con un mayor riesgo a la condición de sobrepeso a los 7 años de edad.
Los diseños panel permiten realizar valoraciones en varias oportunidades de una o más variables cuantitativas y/o cualitativas de los mismos sujetos. La ventaja de este diseño es que permite conocer los cambios individuales y grupales durante el tiempo. Es posible saber los casos específicos que introducen el cambio de la muestra o grupo. En general, durante las valoraciones de los denominados cortes temporales se suele perder muestra (mortalidad) por diversos motivos, disminuyendo los sujetos del estudio a lo largo del tiempo. Una ventaja de estos diseños es que aplican formas mucho más rigurosas de análisis estadístico que los estudios de tendencia y evolución de grupo ${ }^{53}$.

En particular, el diseño de panel mejora las oportunidades para describir las trayectorias del crecimiento y el desarrollo durante el curso de la vida y, además, permite determinar los patrones de las relaciones causales durante períodos de crecimiento físico ${ }^{54}$ más prolongados. Algunos ejemplos de estos estudios son los que buscan determinar el pico de velocidad de crecimiento en niños y adolescentes de una muestra específica, por lo que pueden inferir la velocidad del crecimiento del grupo y de un individuo en particular. Para estimar los patrones de crecimiento individual, los cambios en la tasa de crecimiento y las diferencias en el crecimiento de niños y niñas, se debe desarrollar durante un período un estudio longitudinal. Esto se consigue a través del diseño de Panel. Estos estudios permiten la predicción de diferentes longitudes del cuerpo, siendo posible establecer ecuaciones de regresión lineal para predecir el pico de velocidad de crecimiento de niños y adolescentes; aunque presentan algunas limitaciones, puesto que las diferencias en la sincronización y en el tiempo del crecimiento durante la adolescencia podrían llevar a errores en sus cálculos ${ }^{55}$.

En suma, la complejidad y el elevado costo de los estudios longitudinales y la limitación de los estudios transversales han posibilitado la combinación de ambos métodos para tratar de aprovechar las ventajas de ambos ${ }^{56}$. En este sentido, existe libertad para desarrollar estudios de corte transversal y/o longitudinal, puesto que estas combinaciones son denominadas como estudios mixtos, ya que posibilitan medir muestras de forma transversal en diferentes momentos a lo largo del tiempo. Por lo tanto, son calificados como estudios transversales sucesivos.

Estudios ex post facto: El término ex post facto significa después de ocurridos los hechos. Es un tipo de investigación sistemática en la que el investigador sólo puede registrar las mediciones, no tiene control sobre las variables independientes porque ya ocurrieron los hechos y/o porque son intrínsecamente manipulables ${ }^{57}$, permitiendo observar que ciertos hechos han ocurrido y buscar en el pasado qué factores los han podido ocasionar ${ }^{58}$. En este tipo de estudio, el 
investigador prácticamente se limita a observar, describir, categorizar, simplificar y organizar las variables que configuran el objeto de estudio ${ }^{59}$.

Un ejemplo se puede observar en el estudio efectuado por Argnani y Cols. ${ }^{60}$ donde se evalúa el impacto ambiental sobre el crecimiento humano de niños tibetanos de 8-14 años que nacieron y se criaron por encima de los $4.000 \mathrm{~m}$ en el Himalaya. Otro estudio que permite comprender el impacto de la variable independiente (zona urbana-rural) sobre el crecimiento físico, es el estudio desarrollado por Malina y Cols. ${ }^{61}$, donde se comparó el crecimiento físico de niños de zona urbana y rural del valle de Oaxaca, México.

Una de las desventajas de estos estudios, según lo señalado por Vega ${ }^{58}$, es la imposibilidad de establecer con un margen de seguridad aceptable la causa o causas, debido a la falta de control que se tiene sobre los actores supuestamente causales.

\section{CONCLUSIONES}

Los factores intrínsecos, medioambientales y la interacción entre ambos, son una posibilidad para investigar en diversas poblaciones del mundo, sobre todo porque en la actualidad varios países en Sudamérica se encuentran en un proceso de transición nutricional, con lo que los investigadores deberían abordar y priorizar los posibles factores que podrían afectar el normal crecimiento físico de niños y adolescentes de sus regiones. Por otro lado, la literatura brinda información respecto a los tipos de investigación que se podrían desarrollar en poblaciones en crecimiento y desarrollo; con lo cual es posible diagnosticar, clasificar y monitorizar el crecimiento físico en función del tiempo (transversal y longitudinal) y retrospectiva (ex post facto). Además, es una posibilidad para que los investigadores $\mathrm{y} / \mathrm{o}$ instituciones privadas y gubernamentales puedan promover la investigación y ejercer políticas públicas destinadas a mejorar el estado nutricional de la población, tanto por defecto (hiponutrición) como por sobrealimentación (exceso de peso infantil y juvenil) con el objetivo de disminuir la morbimortalidad asociada a estos dos tipos de trastornos, teniendo en cuenta que, en nuestro entorno son más prevalentes las patologías derivadas del sobrepeso y de la obesidad (diabetes tipo II, accidentes cerebrovasculares, hipertensión arterial, arteriosclerosis, esteatosis hepática, etc.).

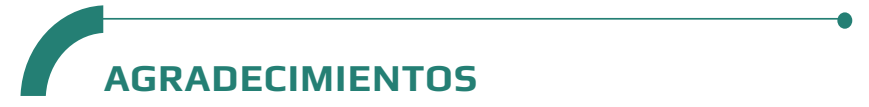

Proyecto Fondecyt 1141295.

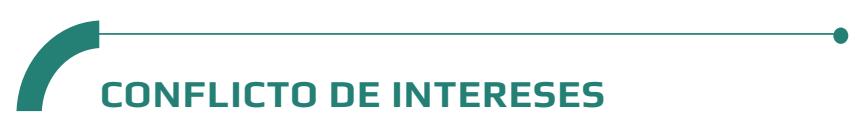

Los autores expresan que no hay conflictos de interés al redactar el manuscrito.

\section{$\longrightarrow$ \\ REFERENCIAS}

(1) Manna PK, De D, Bera TK, Chatterjee K, Ghosh D. Anthropometric Assessment of Physical Growth and Nutritional Status among School Children of North Bengal. Anthropologist. 2011; 13(4): 299-305.

(2) Kuczmarski RJ, Ogden CL, Grummer-Strawn LM, Flegal KM, Guo SS, Wei R, et al. CDC growth charts: United States. Adv Data. 2000; (314): 1-27.

(3) WHO Multicentre Growth Reference Study Group. WHO child growth standards: length/height-for-age, weight-for-age, weight-for-length, weight-for-height and body mass indexfor-age: methods and development. France: World Health Organization; 2006.

(4) de Onis M, Onyango AW, Borghi E, Siyam A, Nishida C, Siekmann ]. Development of a WHO growth reference for school-aged children and adolescents. Bull World Health Organ. 2007; 85(9): 660-7.

(5) Fryar CD, Gu Q, Ogden CL. Anthropometric reference data for children and adults: United States, 2007-2010. Vital Health Stat. 2012; 11(252): 1-48.

(6) World Health Organization. Physical status: the use and interpretation of anthropometry. Report of a WHO Expert Committee. World Health Organ Tech Rep Ser. 1995; 854: 1-452.

(7) Malina RM, Bouchard C. Growth, maturation, and physical activity. Champaign, III, USA: Human Kinetics Books; 1991.

(8) Waltrick AC de A, Duarte M de F da S. Estudo das características antropométricas de escolares de 7 a 17 anos - uma abordagem longitudinal mista e transversal. Rev bras cineantropom desempenho hum. 2000; 2(1): 17-30.

(9) Cameron N. Growth patterns in adverse environments. Am J Hum Biol. 2007; 19(5): 615-21.

(10) Scammon RE. The measurements of the body in childhood. En: Harris JA, Jackson CM, Paterson DG, Scammon RE, editores. 
The measurement of man. Minneapolis, USA: University of Minnesota Press; 1930.

(11) Daza CH. Nutrición infantil y rendimiento escolar. Colomb Med. 1997; 28(2): 92-8.

(12) Cossio-Bolaños MA, de Arruda M, Núñez V, Lancho JL. Efectos de la altitud sobre el crecimiento físico en niños y adolescentes. Rev Andal Med Deporte. 2011; 4(2): 71-6.

(13) Zeferino AMB, Filho AAB, Bettiol $H$, Barbieri MA. Acompanhamento do crescimento. J Pediatr. 2003; 79(Suppl. 1): S23-32.

(14) Keller W. Epidemiología del retraso del crecimiento. En: Retraso del crecimiento lineal en los países en vías de desarrollo. Geneva, Switzerland: Nestlé-Nutrition; 1987.

(15) Cossio-Bolaños M, Figueroa P, Cossio-Bolaños W], Lázari E, Arruda M. Parámetros del crecimiento físico de niños que viven a moderada altitud. Rev Med Hered. 2012; 23(2): 96-106.

(16) Marcondes E. Desenvolvimento da criança: desenvolvimento biológico: crescimento. Rio de Janeiro, Brasil: Sociedade Brasileira de Pediatria; 1994

(17) Westwood M, Kramer MS, Munz D, Lovett JM, Watters GV. Growth and development of full-term nonasphyxiated smallfor-gestational-age newborns: follow-up through adolescence. Pediatrics. 1983; 71(3): 376-82.

(18) Malina RM, Hamill PVV, Lemeshow S. Selected Body Measurements of Children 6-11 Years: United States. Vital Health Stat. 1973; 11(123): 1-48.

(19) Romani S de AM, Lira PIC de. Fatores determinantes do crescimento infantil. Rev Bras Saude Mater Infant. 2004; 4(1): 15-23.

(20) Lavielle-Sotomayor P, Pineda-Aquino V, Jáuregui-Jiménez O, Castillo-Trejo M. Actividad física y sedentarismo: Determinantes sociodemográficos, familiares y su impacto en la salud del adolescente. Rev Salud Pública. 2014; 16(2): 161-72.

(21) Mendonça CP, Anjos LA dos. Aspectos das práticas alimentares e da atividade física como determinantes do crescimento do sobrepeso/obesidade no Brasil. Cad Saúde Pública. 2004; 20(3): 698-709.

(22) García-Ubaque JC, García-Ubaque CA, Vaca-Bohórquez ML. Variables involucradas en la práctica individual y colectiva de hábitos saludables. Rev Salud Pública. 2014; 16(5): 719-32.

(23) Cooper DM. Evidence for and mechanisms of exercise modulation of growth--an overview. Med Sci Sports Exerc. 1994; 26(6): 733-40.

(24) Frisancho AR. Human adaptation: a functional interpretation. Ann Arbor, MI, USA: University of Michigan Press; 1981.

(25) Frisancho AR. Developmental responses to high altitude hypoxia. Am J Phys Anthropol. 1970; 32(3): 401-7.

(26) Frisancho AR. Developmental adaptation to high altitude hypoxia. Int J Biometeorol. 1977; 21(2): 135-46.

(27) Malina RM. Growth and development: the first twenty years in man. Minneapolis, USA: Burgess Pub. Co.; 1975.

(28) van Wieringen JC. Secular growth changes. En: Human growth: a comprehensive treatise. 2nd ed. New York: Plenum Press; 1986. p. 307-15.
(29) Ljung BO, Bergsten-Brucefors A, Lindgren G. The secular trend in physical growth in Sweden. Ann Hum Biol. 1974; 1(3): 245-56.

(30) Wedderkopp N, Froberg K, Hansen HS, Andersen LB. Secular trends in physical fitness and obesity in Danish 9-year-old girls and boys: Odense School Child Study and Danish substudy of the European Youth Heart Study. Scand J Med Sci Sports. 2004; 14(3): 150-5.

(31) Matton L, Duvigneaud N, Wijndaele K, Philippaerts R, Duquet $W$, Beunen $G$, et al. Secular trends in anthropometric characteristics, physical fitness, physical activity, and biological maturation in Flemish adolescents between 1969 and 2005. Am J Hum Biol. 2007; 19(3): 345-57.

(32) Tanner JM. The Trend Towards Earlier Physical Maturation. En: Meade JE, Parkes AS, editores. Biological Aspects of Social Problems. New York, USA: Springer US; 1965. p. 40-65.

(33) Eveleth PB, Tanner JM. Worldwide variation in human growth. Cambridge [England]; New York: Cambridge University Press; 1976.

(34) Tanner JM. A history of the study of human growth. Cambridge [England]; New YorkSecular growth changes in Europe: Cambridge University Press; 1981.

(35) Bodzsaŕ ÉB, Susanne C. Secular growth changes in Europe. Budapest: Eötvös University Press; 1998.

(36) Rebato E. Crecimiento: una visión desde la Antropología Física. Rev Esp Antrop Fís. 2010; (31): 85-110.

(37) Saranga S, Maia J, Rocha J, Nhantumbo L, Prista A. Crescimento somático na população africana em idade escolar: Estado actual do conhecimento. Rev Port Cien Desp. 2006; 6(1): 81-93.

(38) Morrow JR, Jackson AW, Disch JG, Mood DP. Measurement and evaluation in human performance. 2nd ed. Champaign, IL: Human Kinetics; 2000.

(39) Hamill PV, Drizd TA, Johnson CL, Reed RB, Roche AF. NCHS growth curves for children birth-18 years. United States. Vital Health Stat. 1977; 11(165): i-iv, 1-74.

(40) Rodríguez G, Gallego S, Fleta J, Moreno LA. Uso del índice de masa corporal para valorar la obesidad en niños y adolescentes. Rev Esp Obes. 2006; 4(5): 284-8.

(41) Grupo de trabajo de la Guía de Práctica Clínica sobre la Prevención y el Tratamiento de la Obesidad Infantojuvenil. Guía de Práctica Clínica sobre la Prevención y el Tratamiento de la Obesidad Infantojuvenil. Plan de Calidad para el Sistema Nacional de Salud del Ministerio de Sanidad y Política Social. Agència d’Avaluació de Tecnologia i Recerca Mèdiques. Centro Cochrane Iberoamericano, coordinador. Barcelona: Ministerio de Ciencia e Innovación; 2009. (Guías de Práctica Clínica en el SNS: AATRM № 2007/25).

(42) Cole TJ, Bellizzi MC, Flegal KM, Dietz WH. Establishing a standard definition for child overweight and obesity worldwide: international survey. BMJ. 2000; 320(7244): 1240-3.

(43) Hormiguera $X$. Circunferencia de la cintura y riesgo cardiovascular en población española. Clin Invest Arterioscl. 2007; 19(2): 90-1.

(44) Hennekens $\mathrm{CH}$, Buring JE. Epidemiology in medicine. 1st ed. Mayrent SL, editor. Boston, USA: Little, Brown; 1987. 
(45) Marques RM, Marcondes E, Berquó E, Prandi R, Nunes J. Crescimento e Desenvolvimento Pubertário em Crianças e Adolescentes Brasileiros: II - Altura e Peso. São Paulo, Brasil: Brasileira de Ciências; 1982.

(46) Conde WL, Monteiro CA. Body mass index cutoff points for evaluation of nutritional status in Brazilian children and adolescents. J Pediatr. 2006; 82(4): 266-72.

(47) Zeger SL, Liang KY. An overview of methods for the analysis of longitudinal data. Stat Med. 1992; 11(14-15): 1825-39.

(48) Edwards L. Modern statistical techniques for the analysis of longitudinal data in biomedical research. Pediatr Pulmonol. 2000; 30(4): 330-44.

(49) Firebaugh G. Analyzing repeated surveys. Thousand Oaks, CA, USA: Sage Publications; 1997.

(50) Hauspie RC, Vercauteren M, Susanne C. Secular changes in growth and maturation: an update. Acta Paediatr Suppl. 1997; 423: 20-7.

(51) Kuri P. Impacto de los estudios longitudinales en la práctica de la salud pública. Salud Pública Méx. 2007; 49(sup 1): 23-4.

(52) Stettler N, Zemel BS, Kumanyika S, Stallings VA. Infant weight gain and childhood overweight status in a multicenter, cohort study. Pediatrics. 2002; 109(2): 194-9.

(53) Ávila HL. Introducción a la metodología del la investigación. Chihuahua, México: Ediciones Eumed; 2006.
(54) Blossfeld H-P, Schneider T, Doll J. Methodological Advantages of Panel Studies: Designing the New National Educational Panel Study (NEPS) in Germany. JERO. 2009; 1(1): 10-32.

(55) Malina RM, Claessens AL, Van Aken K, Thomis M, Lefevre J, Philippaerts $R$, et al. Maturity offset in gymnasts: application of a prediction equation. Med Sci Sports Exerc. 2006; 38(7): 1342-7.

(56) Argente J. Curvas de crecimiento en España: ¿cuál es el estado actual? Pediatr Integral. 2009; XIII(1): 77-80.

(57) Kerlinger FN. Foundations of behavioral research. 2nd ed. Delhi: Surjeet Publications; 1983.

(58) Vega C. Papel de trabajo, Aspectos epistemológicos de la estimación estadística de modelos: Investigación Ex-postFacto. 2015.

(59) Mateo J. La investigación ex post-facto. En Barcelona, España: Universitat oberta de Catalunya; 2000.

(60) Argnani L, Cogo A, Gualdi-Russo E. Growth and nutritional status of Tibetan children at high altitude. Coll Antropol. 2008; 32(3): 807-12.

(61) Malina RM, Himes JH, Stepick CD, Lopez FG, Buschang PH. Growth of rural and urban children in the valley of Oaxaca, Mexico. Am J Phys Anthropol. 1981; 55(2): 269-80. 